\title{
PROBING THE LARGE-SCALE TOPOLOGY OF THE HELIOSPHERIC MAGNETIC FIELD USING JOVIAN ELECTRONS
}

\author{
M. J. OWens ${ }^{1,3}$, T. S. Horbury ${ }^{1}$, And C. N. ArgE ${ }^{2}$ \\ ${ }^{1}$ Space and Atmospheric Physics, Imperial College, London SW7 2AZ, UK \\ 2 Air Force Research Laboratory/Space Vehicles Directorate, Kirtland Air Force Base, NM 87117, USA \\ Received 2009 September 16; accepted 2010 March 20; published 2010 April 21
}

\begin{abstract}
Jupiter's magnetosphere acts as a point source of near-relativistic electrons within the heliosphere. In this study, three solar cycles of Jovian electron data in near-Earth space are examined. Jovian electron intensity is found to peak for an ideal Parker spiral connection, but with considerable spread about this point. Assuming the peak in Jovian electron counts indicates the best magnetic connection to Jupiter, we find a clear trend for fast and slow solar wind to be over- and under-wound with respect to the ideal Parker spiral, respectively. This is shown to be well explained in terms of solar wind stream interactions. Thus, modulation of Jovian electrons by corotating interaction regions (CIRs) may primarily be the result of changing magnetic connection, rather than CIRs acting as barriers to cross-field diffusion. By using Jovian electrons to remote sensing magnetic connectivity with Jupiter's magnetosphere, we suggest that they provide a means to validate solar wind models between 1 and $5 \mathrm{AU}$, even when suitable in situ solar wind observations are not available. Furthermore, using Jovian electron observations as probes of heliospheric magnetic topology could provide insight into heliospheric magnetic field braiding and turbulence, as well as any systematic under-winding of the heliospheric magnetic field relative to the Parker spiral from footpoint motion of the magnetic field.
\end{abstract}

Key words: interplanetary medium - solar wind - Sun: heliosphere - Sun: magnetic topology - Sun: particle emission

Online-only material: color figures

\section{INTRODUCTION}

Jupiter's magnetosphere is a major source of relativistic electrons in the heliosphere (Teegarden et al. 1974; Chenette et al. 1974; Mewaldt et al. 1976). Pioneer 10 observed a steady increase in the $\sim 1 \mathrm{MeV}$ electron flux as it approached Jupiter (Pyle \& Simpson 1977), though there was significant modulation associated with corotating interaction regions (CIRs; e.g., Gosling \& Pizzo 1999, and references therein) in the solar wind. Jovian electrons have been found throughout the inner heliosphere, propagating into less than 0.5 AU (Eraker \& Simpson 1979) and out to at least $11 \mathrm{AU}$ (Hamilton \& Simpson 1979). Voyager 1 and Voyager 2 (Schardt et al. 1983), and more recently Ulysses (Simpson et al. 1993; Heber et al. 2007) observations, have been able to investigate the variation in energetic electron intensity with both increasing radial and latitudinal extent from the Jovian source. Thus, this point source of relativistic electrons in the heliosphere has proved to be an invaluable test bed for energetic particle propagation models and diffusion theory (e.g., Conlon \& Simpson 1977; Ferreira et al. 2001a, 2001b).

In near-Earth space, Jovian electrons display a strong 13month periodicity, associated with Jupiter's synodic period (Chenette 1980; Moses 1987). On shorter timescales during periods of solar minimum, relativistic electron intensities have a clear 27-day modulation. It is unclear whether this is the result of density enhancements in CIRs acting as barriers to cross-field diffusion in interplanetary space (Conlon 1978), CIRs at Jupiter stimulating electron release from the magnetosphere (Chenette 1980; Morioka \& Tsuchiya 1996; Tsuchiya et al. 1999), or because of changing magnetic connectivity to Jupiter (Moses 1987, and investigated further in this study). Similarly, interest

\footnotetext{
3 Now at: Space Environment Physics Group, Department of Meteorology, University of Reading, P.O. Box 243, Reading, RG6 6BB, UK.
}

in Jovian electrons comes from a number of areas: from their use as a remote probe of the Jovian magnetosphere, as a test of scattering and energetic particle transport, and even as a possible source of energetic electrons in the terrestrial magnetosphere (Baker et al. 1979), though this latter idea has now largely been discounted (Christon et al. 1989). This study, however, focuses on what can be learned about the underlying structure of the heliospheric magnetic field by using Jovian electrons as tracers of flux tubes which connect the observer to Jupiter's magnetosphere.

\section{DATA}

The Cosmic Ray Nuclear Composition (CRNC) instrument (Chenette 1980) on the IMP 8 spacecraft observed Jovian electrons predominantly in the ID3 energy channel, which covered a range from 2 to $12 \mathrm{MeV}$. Jovian electrons generally exhibit less steep energy spectra than solar electron bursts (e.g., Mewaldt et al. 1976; Lheureux \& Meyer 1976), meaning the ID2 energy channel, covering $0.7-2 \mathrm{MeV}$, can be used to discriminate between energetic electrons of Jovian and solar origins. On this basis, Chenette (1980) selected periods of quiet solar activity using a ratio of the electron count rate in the two CRNC energy channels, given by $0.5<$ ID2/ID3 $<2.5$. During such intervals, electrons are assumed to be predominantly of Jovian origin, with some contribution from galactic cosmic rays. We assume that galactic cosmic ray variations do not systematically vary over the Jovian synodic period.

In this study, we use the same Chenette (1980) method to produce a $1 \mathrm{hr}$ resolution time series of Jovian electron count rates in near-Earth space spanning the whole of the CRNC data set (available at http://ulysses.sr.unh.edu/WWW/Simpson/ imp8.html), which covers the years 1974 through 2001. As we are only interested in the variation of Jovian electron counts 


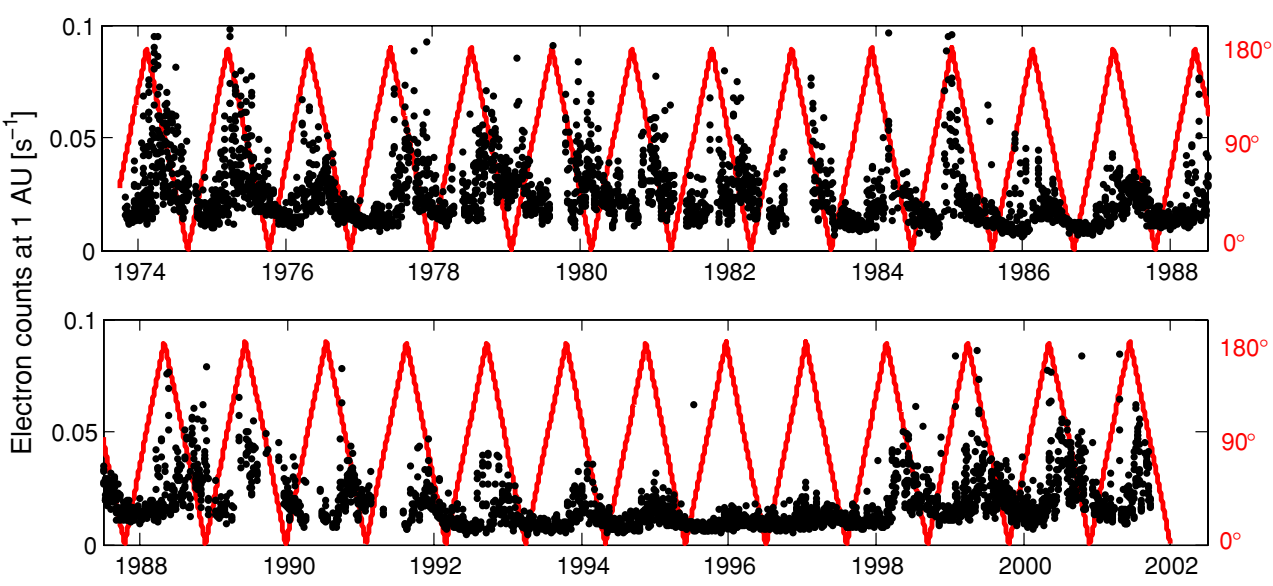

Figure 1. Overview of the Jovian electron counts measured in near-Earth space using the CRNC instrument aboard the IMP 8 spacecraft. The data have been averaged from hourly to daily values for the purposes of clarity. The red line (and left-hand axis) shows the Earth-Sun-Jupiter angle, with $0^{\circ}\left(180^{\circ}\right)$ corresponding to Jovian conjunction (opposition).

(A color version of this figure is available in the online journal.)
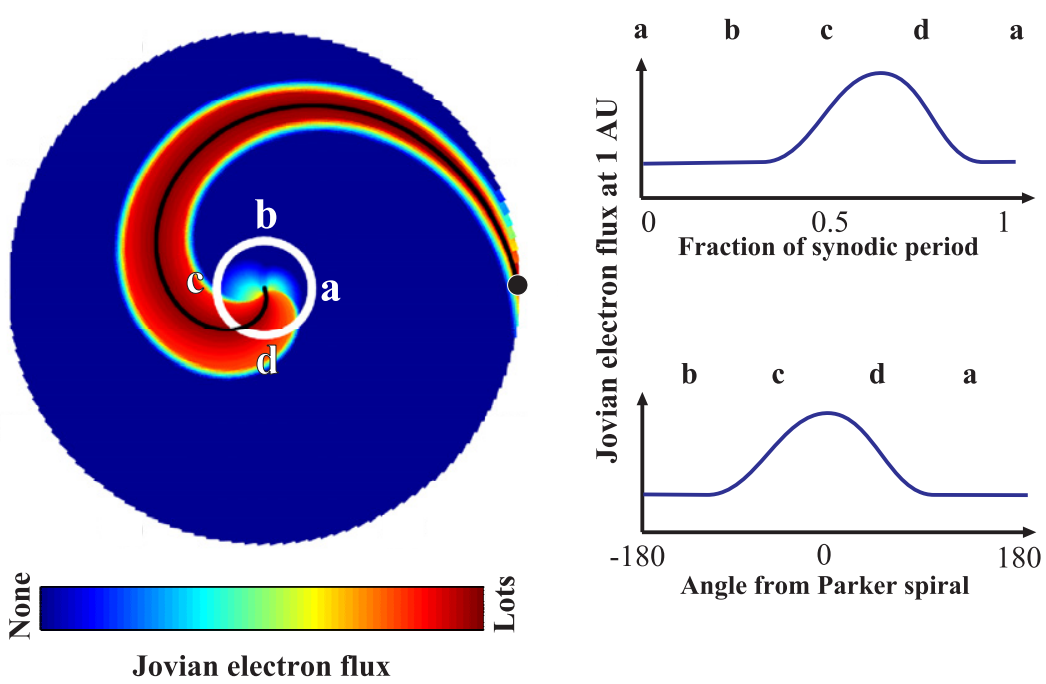

Figure 2. Left: a sketch of Jovian electron flux in the ecliptic plane, assuming electrons can move freely along the magnetic field and diffuse slowly perpendicular to the field. A Parker spiral magnetic field with a constant solar wind speed of $450 \mathrm{~km} \mathrm{~s}^{-1}$ was used. The position of Jupiter is shown as the solid black circle, while the white ring indicates Earth's orbit. Thus, position "a" corresponds to conjunction, whereas "c" is opposition. Right: the expected 1 AU fluxes as a function of Jovian synodic period (top) and separation from the Parker spiral connection (bottom).

(A color version of this figure is available in the online journal.)

over intervals of one Jovian synodic period $\left(T_{\mathrm{SYN}} \sim 399\right.$ days $)$ or less, it is not necessary to account for instrumental effects and convert the data from counts to differential energy flux.

Solar wind magnetic field and plasma data in near-Earth space are provided by the OMNI data set (available from the NSSDC: http://omniweb.gsfc.nasa.gov/). In the analysis that follows, only periods for which there is at least $30 \%$ daily coverage in both the solar wind and Jovian electron data are used.

Figure 1 shows an overview of the resultant CRNC Jovian electron data set, with the $1 \mathrm{AU}$ electron counts shown in black. For clarity, the time series has been averaged from 1 $\mathrm{hr}$ to daily values. The red line and right-hand axis show the Earth-Sun-Jupiter angle. The electron counts are clearly organized by the synodic period, but with a phase shift: the peak in counts does correspond with Earth-Jupiter conjunction, and the minima in counts are offset from opposition. This is due to magnetic connection between Earth and Jupiter ordering the near-Earth Jovian electron flux (Chenette 1980), as discussed in the following section.

\section{MAGNETIC CONNECTIVITY WITH JUPITER}

The left panel of Figure 2 shows a sketch of Jovian electron flux in the ecliptic plane, assuming electrons can move freely along the magnetic field and diffuse slowly perpendicular to the field. A Parker spiral magnetic field with a constant solar wind speed of $450 \mathrm{~km} \mathrm{~s}^{-1}$ was used. The position of Jupiter is shown as the solid black circle, while the white ring indicates Earth's orbit. Thus, position "a" corresponds to conjunction, whereas "c" is opposition. The top-right panel shows the expected 1 AU Jovian electron flux as a function of time. The sketched configuration shows 1 AU Jovian electron flux peaking just after Earth has passed opposition, in approximate agreement with the observed Jovian electron "season" during the latter half of the Jovian synodic period, with a peak in counts at approximately $0.6 T_{\mathrm{SYN}}$ (Chenette 1980, see also Figure 1 and the right-hand panel of Figure 3). This corresponds to an inecliptic Earth-Sun-Jupiter angle of $\sim 220^{\circ}$, measured in the direction of solar rotation (see also Figure 4). The Parker spiral 


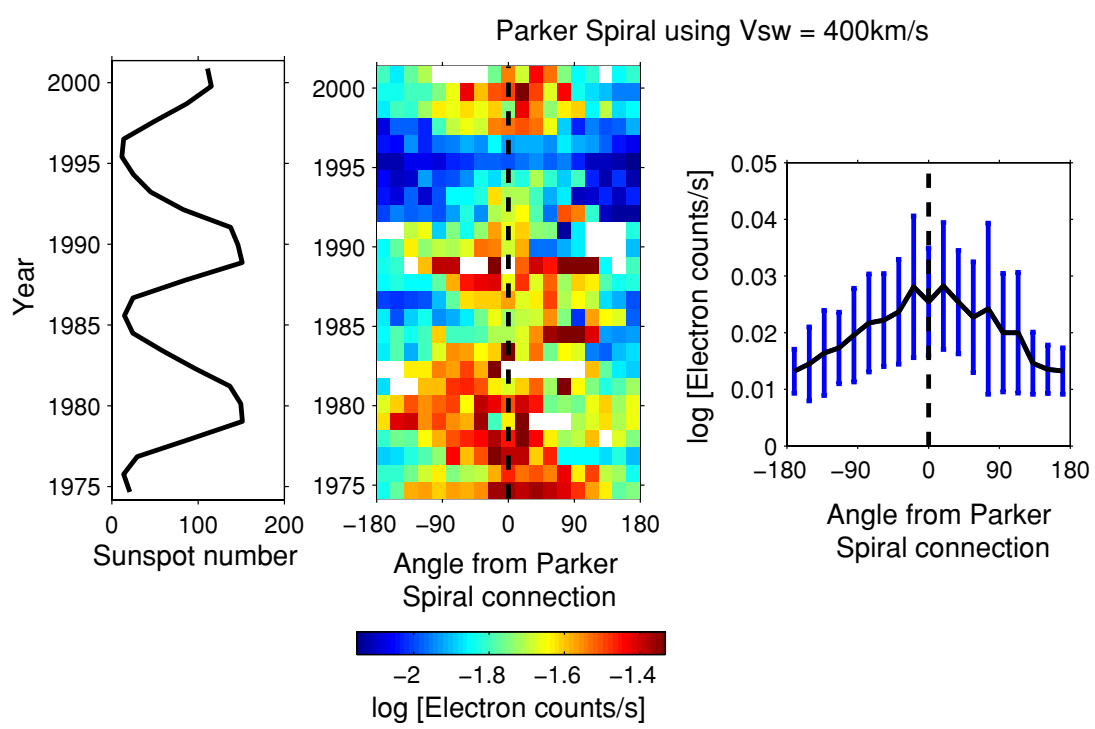

Figure 3. Summary of Jovian electron counts in near-Earth space over the last three solar cycles. Left: sunspot number. Center: Jovian electron counts as a function of time and angular separation from magnetic connection to Jupiter $\left(\Delta \phi_{\mathrm{PS}}\right)$, assuming a perfect Parker spiral and a solar wind speed of $400 \mathrm{~km} \mathrm{~s}{ }^{-1}$. A logarithmic color scale is used. Right: a superposed epoch analysis of the same data. Jovian electron counts are centered on an ideal Parker spiral connection, but there is considerable spread in the data.

(A color version of this figure is available in the online journal.)

angle of the magnetic field to the radial direction, $\gamma$, at any distance, $R$, in the solar equatorial plane is given by

$$
\tan \gamma=\frac{\Omega R}{V_{\mathrm{SW}}}
$$

where $V_{\mathrm{SW}}$ is the solar wind speed and $\Omega$ is the solar rotation speed (i.e., $2 \pi / T_{\mathrm{SID}}$, where $T_{\mathrm{SID}}$ is the solar sidereal rotation period, $\sim 25$ days). If the solar wind speed is assumed to be constant, then $\phi$, the angular separation of Earth from an ideal Parker spiral magnetic field connection at $R$, is simply given by

$$
\phi=\frac{\Omega}{V_{\mathrm{SW}}}(R-1 \mathrm{AU}) .
$$

Thus, an angular separation of $220^{\circ}$ between 1 and $5.2 \mathrm{AU}$ corresponds to an ideal Parker spiral magnetic connection for nominal solar wind speeds (e.g., $\sim 400 \mathrm{~km} \mathrm{~s}^{-1}$ ).

The center panel of Figure 3 shows the near-Earth Jovian electron data from Figure 1 as a function of angular separation from magnetic connection to Jupiter, assuming a perfect Parker spiral and a constant solar wind speed of $400 \mathrm{~km} \mathrm{~s}^{-1}$. The data are separated into stack plots of Jovian synodic periods, increasing in time up the $y$-axis. A logarithmic color scale is used. The left panel shows the corresponding sunspot number. There is some evidence for a solar cycle variation in the data, with higher counts being associated with solar maximum (e.g., Henize et al. 2003, and references therein). A drop in nearEarth Jovian electron flux during 1995-1996, the previous solar minimum, is immediately obvious. This has been attributed to either a change in the Jovian source strength or a softening of the Jovian electron energy spectrum, but is unlikely to be the result of changing interplanetary propagation (Kanekal et al. 2003).

The right-hand panel of Figure 3 shows the observations in the form of a superposed epoch plot of each synodic period, equivalent to the sketched flux in the top-right panel of Figure 2. The Jovian electron "season" can clearly be seen during the latter half of the Jovian synodic period, with a peak in counts at approximately $0.6 T_{\mathrm{SYN}}$. The ordering of Jovian electron counts with a Parker spiral and a constant solar wind speed results in a fairly broad peak. While this could be due to large crossfield diffusion, it is more likely the result of solar wind speed variations changing the angular separation which results in magnetic connection between Earth and Jupiter.

To further investigate this issue, the near-Earth solar wind speed from the OMNI data is used to compute $\Delta \phi_{\mathrm{PS}}$, the angular separation of Jupiter from an ideal Parker spiral connection to Earth, for each CRNC measurement. This angle is shown in Figure 4: it can vary considerably from fast (red) to slow (blue) solar wind streams. We also attempt to remove any long-term variations resulting from either changes in the Jovian electron source (Henize et al. 2003) and/or changes in the sensitivity of the detector from gradual instrumental degradation, thus isolating the effect of electron propagation from Jupiter to Earth. To this end, electron counts for each synodic period (399 days) are individually normalized to the maximum/minimum values within that synodic period.

The left panels in Figure 5 show synodic-period stack plots of the normalized electron counts as a function of $\Delta \phi_{\mathrm{PS}}$. Note that by normalizing the data, the dramatic change in Jovian electron behavior during 1995-1996 has been effectively removed, in agreement with it being a change in the Jovian source rather than an effect of interplanetary propagation (Kanekal et al. 2003). The superposed epoch plot shows that the ordering of Jovian electrons about the expected Parker spiral connection is more apparent than in the unnormalized data, but considerable spread remains. Again, one possible explanation for this broad peak is efficient cross-field diffusion, but alternatives are investigated in the remainder of this study.

The center and right-hand panels of Figure 5 show the normalized data split into slow $\left(<400 \mathrm{~km} \mathrm{~s}^{-1}\right)$ and fast $\left(>450 \mathrm{~km} \mathrm{~s}^{-1}\right)$ solar wind, respectively. Assuming the peak in the electron counts indicates the best magnetic connection of Earth to Jupiter, the magnetic field associated with slow wind is strongly underwound compared to the ideal Parker spiral, whereas fast wind is over-wound. 


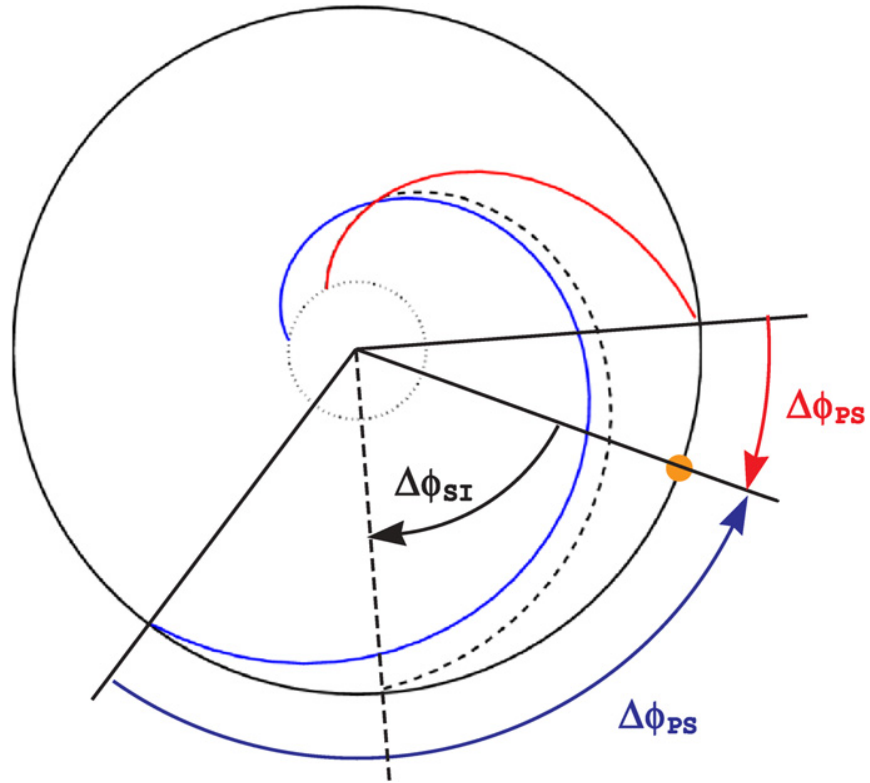

Figure 4. Sketch of magnetic connectivity between Earth and Jupiter in the heliographic equatorial plane. The red (blue) curve shows an ideal Parker spiral magnetic field line between 1 and 5 AU for typical fast (slow) solar wind of $800(300) \mathrm{km} \mathrm{s}^{-1}$. The orange dot shows the position of Jupiter. The angular separation from the ideal Parker spiral magnetic field is denoted by $\Delta \phi_{\mathrm{PS}}$. Interaction between fast and slow streams causes a deflection of the fields from the ideal Parker spiral, with the resultant field shown as the black dashed line. The angular separation of Jupiter from the stream-interacting field line is denoted by $\Delta \phi_{\mathrm{SI}}$. The magnetic field in slow (fast) solar wind becomes under(over-)wound compared to the ideal Parker Spiral.

(A color version of this figure is available in the online journal.)

The top panel of Figure 6 shows Jovian electron counts as a function of both the near-Earth solar wind speed and $\Delta \phi_{\mathrm{PS}}$, the angular separation from the ideal Parker spiral. The data set was divided into $10^{\circ}$ bins in $\Delta \phi_{\mathrm{PS}}$. In solar wind speed, the bulk of the data set was split into $20 \mathrm{~km} \mathrm{~s}^{-1}$ bins, though at high and low solar wind speeds it was necessary to use larger bin widths to obtain adequate statistics. Assuming again that Jovian electron counts are maximized at the optimum magnetic connection to Jupiter, there is a clear trend for the magnetic field threading slow solar wind to be under-wound with respect to an ideal Parker spiral, while fast wind is over-wound, with no net deviation occurring close to $430 \mathrm{~km} \mathrm{~s}^{-1}$. The linear best fit (not shown) is given by

$$
\begin{aligned}
& \text { Deviation from ideal Parker spiral (deg) } \\
& \quad=208^{\circ}-0.394 V_{\mathrm{SW}}\left(\operatorname{deg~s~km}{ }^{-1}\right) .
\end{aligned}
$$

The reason for this speed-dependent deviation from the ideal Parker spiral is likely the result of solar wind stream interactions, shown schematically in Figure 4. If we assume an in-ecliptic bulk solar wind speed of $V_{0}$ and that solar wind at speeds above/ below this value is subject to a force analogous to aerodynamic drag (e.g., Cargill 2004), then

$$
\frac{d V}{d t}=-C\left(V-V_{0}\right)\left|V-V_{0}\right|,
$$

where $V(R)$ is the solar wind speed at $R$ and $C$ is the aerodynamic drag constant. By integrating this equation between $R=1$ and $5 \mathrm{AU}$, it is possible to compute the transit time and the angle between magnetic connection and an ideal Parker spiral (i.e., when $V$ is constant with $R$ ). The black line in the top panel of Figure 6 shows the best fit of this function to the observed peak in Jovian electron counts. It uses parameters $V_{0}=400 \mathrm{~km} \mathrm{~s}^{-1}$ and $C=1 \times 10^{-6} \mathrm{~km}^{-1}$. Solar wind stream interactions are further investigated by a detailed analysis of a shorter time period in the following section.

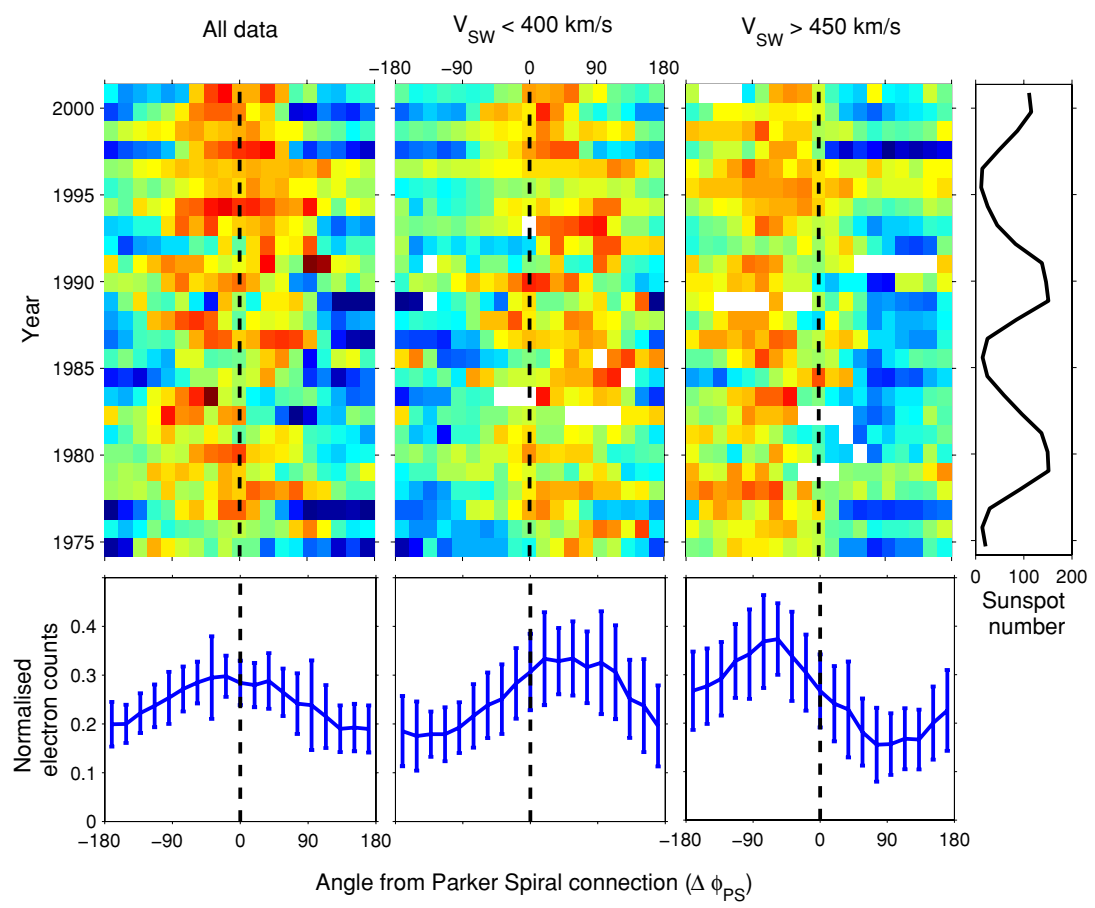

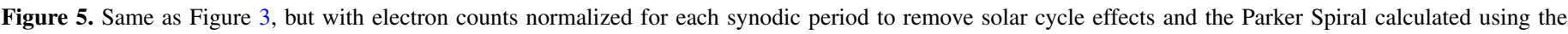

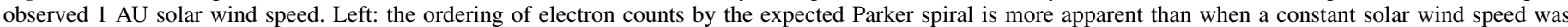

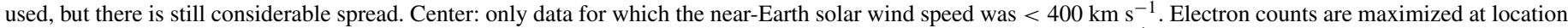

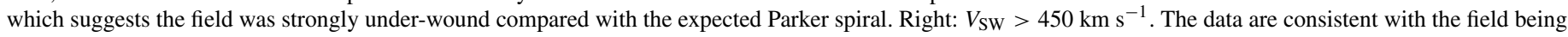
over-wound compared to an ideal Parker spiral.

(A color version of this figure is available in the online journal.) 


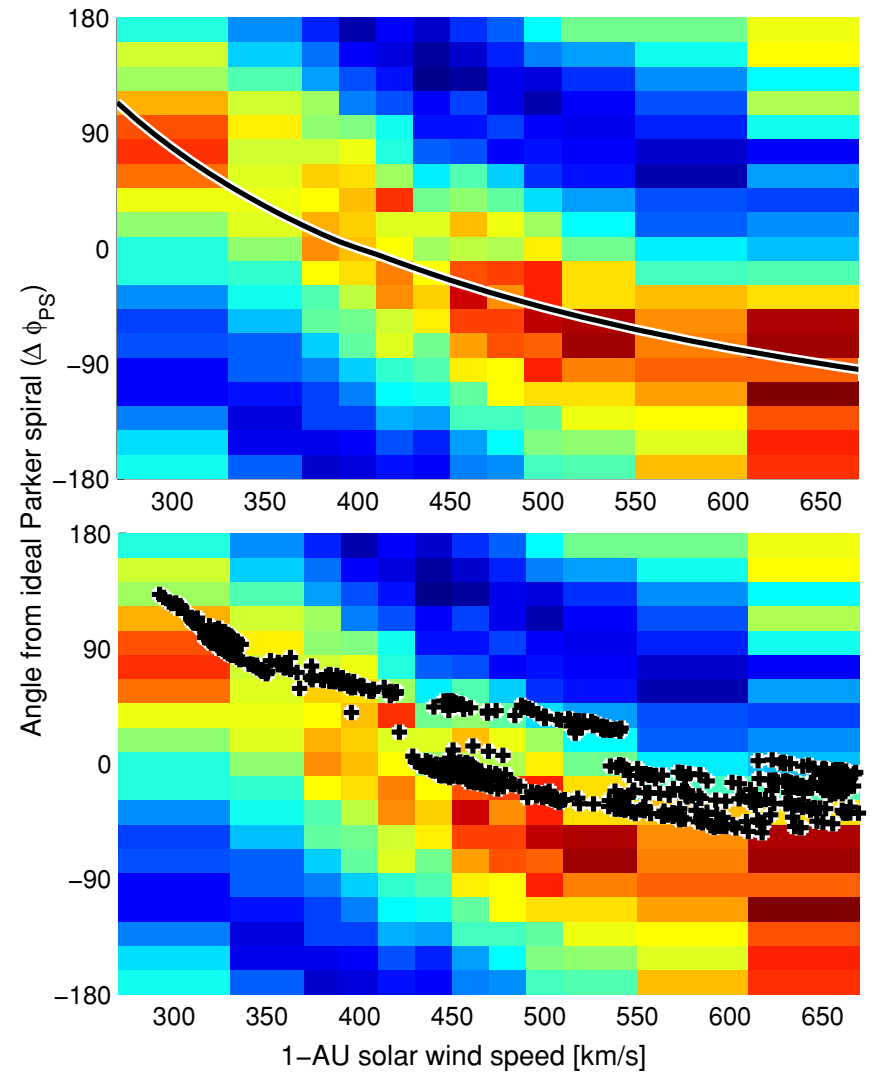

Figure 6. Normalized Jovian electron counts shown as a function of the nearEarth solar wind speed and angular separation from the ideal Parker spiral connection. Assuming electron counts are maximized at the true magnetic connection to Jupiter, there is a clear trend for slow solar wind to be underwound with respect to an ideal Parker spiral, while fast wind is over-wound, with no net deviation occurring close to $400 \mathrm{kms} \mathrm{s}^{-1}$. Top panel: the best fit to the peak Jovian electron intensity is shown as the black line. Bottom panel: the black crosses show the difference between stream-interacting and the ideal Parker spiral magnetic connectivity to Jupiter (i.e., $\Delta \phi_{\mathrm{SI}}-\Delta \phi_{\mathrm{PS}}$ ) as a function of near-Earth solar wind speed for CR1618. The change in magnetic connectivity introduced by stream interactions appears to qualitatively agree with the magnetic connection suggested by the Jovian electron observations.

(A color version of this figure is available in the online journal.)

\section{RECURRENT SOLAR WIND STREAMS OF 1974}

During mid-to-late 1974, the in-ecliptic solar wind conditions were particularly stable from one Carrington rotation to the next (e.g., Zieger \& Hansen 2008). At this time, Pioneer 11 was upstream of Jupiter, providing in situ measurements of the solar wind conditions. This period has been studied by Conlon \& Simpson (1977), who associated periodic variations in the near-Jupiter electron flux with CIR-associated density structures acting as a barrier between the source and the observer. Here, we examine the interplanetary magnetic field structure between Earth and Jupiter for comparison with the near-Earth Jovian electrons.

Carrington rotations 1618 and 1619, covering from 1974 August to October, had particularly stable solar wind structures both near Earth and at Pioneer 11's orbit. As there are little CRNC data for CR1619, CR1618 is used exclusively here. The top panel of Figure 7 shows the near-Earth solar wind speed for CR1618, with inward, outward, and undetermined magnetic sectors shown as blue, red, and black dots, respectively. The solar wind at this time exhibited a two-stream and two-sector structure, characteristic of solar minimum (e.g., McComas et al. 2003). The large dots in the bottom panel of Figure 7 show the

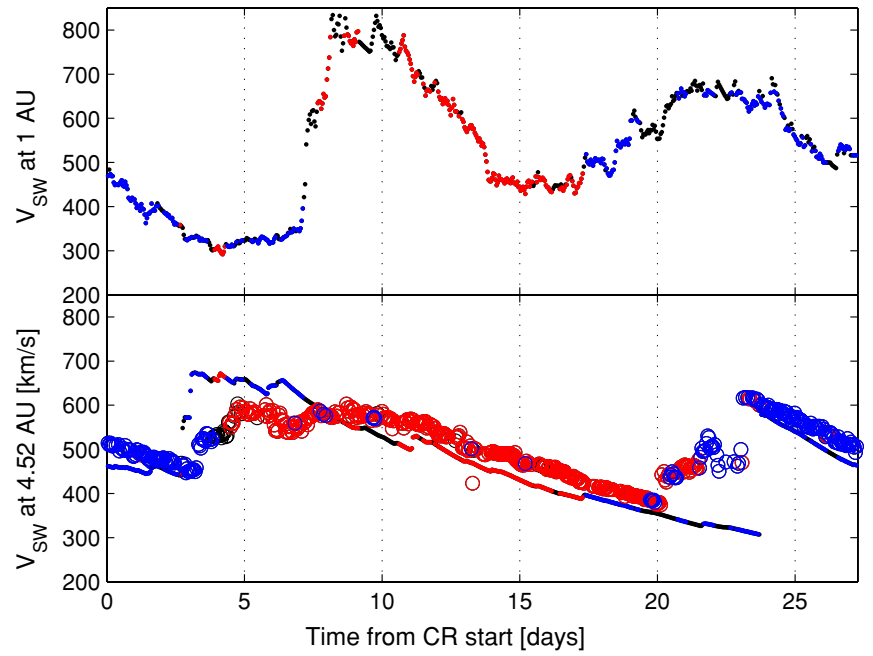

Figure 7. Solar wind speed during Carrington rotation 1618 (1974 August/ September), with inward, outward, and undetermined magnetic sectors shown as blue, red, and black dots, respectively. Top panel: near-Earth observations. Bottom panel: the large dots show observations from Pioneer 11 which was upstream of Jupiter, while the smaller dots show the near-Earth solar wind propagated to Pioneer 11's location using a simple ballistic scheme which allows for stream interaction.

(A color version of this figure is available in the online journal.)

Pioneer 11 observations just upstream of Jupiter at $4.52 \mathrm{AU}$, in the same format. Again, the solar wind shows a two-stream and two-sector structure, though the variation in solar wind speed between the fast and slow streams is reduced. The smaller dots show near-Earth solar wind propagated out to Pioneer 11's location using the Arge \& Pizzo (2000) technique, which ballistically maps the solar wind radially outward, but allows for stream interactions. This model is briefly described below. Note that this model is used here for demonstration purposes, rather than as a validation of the model itself.

Each solar wind packet (in this study, $1 \mathrm{hr}$ of data, corresponding to $\sim 1^{\circ}$ of heliolongitude) is mapped radially forward at constant speed for a time $d t$. In this study we use $d t=1 \mathrm{hr}$. If a solar wind packet catches up with the packet ahead of it, they are not allowed to pass each other, but instead move together at the average speed of the two packets, as shown as the dashed line in Figure 4. While such a scheme oversimplifies the complex, three-dimensional interaction between solar wind streams (Pizzo 1991), it has been shown to work well for propagating predicted solar wind speeds from the corona to Earth (Arge \& Pizzo 2000; Owens et al. 2005, 2008). As can be seen from the bottom panel of Figure 7, for quasi-stable solar wind structures, it also captures the large-scale stream structure between the Earth and the Jupiter. The magnetic sectors obtained from this propagation model are also in general agreement with the observations, though there are timing differences in the sector crossings, which may be the result of the slight heliographic latitudinal offset between Earth and Pioneer 11 at this time (the propagation routine is one dimensional, which leads to a purely two-dimensional magnetic field structure).

Figure 8 shows the magnetic field connectivity between 1 and 5 AU obtained from applying the solar wind propagation procedure to the observed $1 \mathrm{AU}$ solar wind speeds during CR1618. Colored lines denote magnetic sector structure in the same format as Figure 7. Carrington longitudes at $1 \mathrm{AU}$ are found to typically connect to longitudes at $5 \mathrm{AU}$ which are approximately $200^{\circ}$ behind with respect to Earth's orbital 


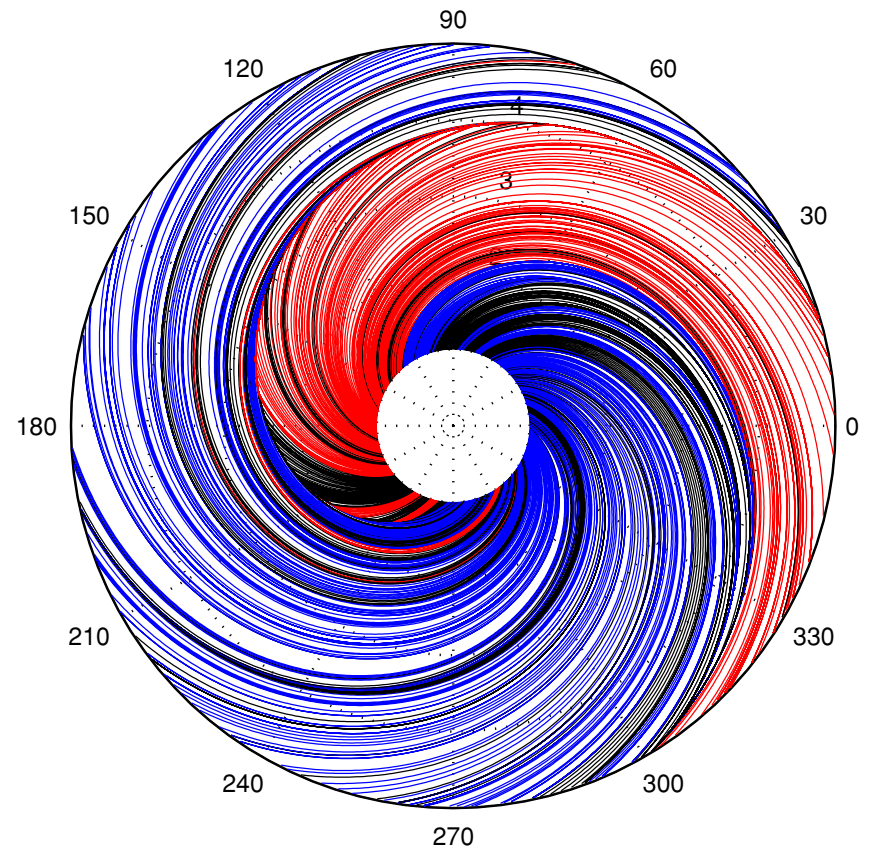

Figure 8. Plot of the magnetic field connectivity from 1 to $5 \mathrm{AU}$ in the heliographic equatorial plane, obtained from propagating the observed $1 \mathrm{AU}$ solar wind speed during Carrington rotation 1618. Red/blue/black lines show outward-/inward-/undetermined- polarity magnetic sectors, respectively. This magnetic configuration takes into account the effect of solar wind stream interaction and can be used to compute $\Delta \phi_{\mathrm{SI}}$.

(A color version of this figure is available in the online journal.)

direction, though there is a significant amount of variation in this value. This predicted magnetic field structure can be used to calculate an angular magnetic separation between Earth and Jupiter, $\Delta \phi_{\mathrm{SI}}$, which unlike $\Delta \phi_{\mathrm{PS}}$ used in the previous section, takes the effect of solar wind stream interactions into account. This angle is also defined in Figure 4.

The top panel of Figure 9 shows how the observed nearEarth Jovian electron counts during CR1618, indicated by black crosses and the right-hand axis, vary with estimates of magnetic connectivity to Jupiter. The blue points and the left-hand axis show $\Delta \phi_{\mathrm{PS}}$, the angular separation of Earth from magnetic connection to Jupiter estimated when solar wind stream interactions are not taken into account, while the blue points show $\Delta \phi_{\mathrm{SI}}$, where stream interactions are included. The bottom panels show the corresponding scatter plots. Stream interactions lead to a reduction in the variability in magnetic connectivity, as they lead to an equilibration of solar wind speeds toward the mean value. The significance of the correlation between Jovian electron counts and Jovian magnetic connectivity increases marginally when stream interactions are included: the Spearman rank-ordered correlation coefficient (Press et al. 1989), which tests the significance of a correlation rather than its linearity, increases from 0.720 to 0.743 for the binned CR1618 data.

The color map in the bottom panel of Figure 6 shows three solar cycles of near-Earth Jovian electron counts sorted by nearEarth solar wind speed and $\Delta \phi_{\mathrm{PS}}$, the angular separation from an ideal Parker Spiral connection to Jupiter. Overplotted as black crosses are the differences between model-inferred and the ideal Parker spiral connectivity to Jupiter (i.e., $\Delta \phi_{\mathrm{SI}}-\Delta \phi_{\mathrm{PS}}$ ) as a function of near-Earth solar wind speed for CR1618. There is qualitative agreement between the change in magnetic connectivity introduced by stream interactions and the peak in
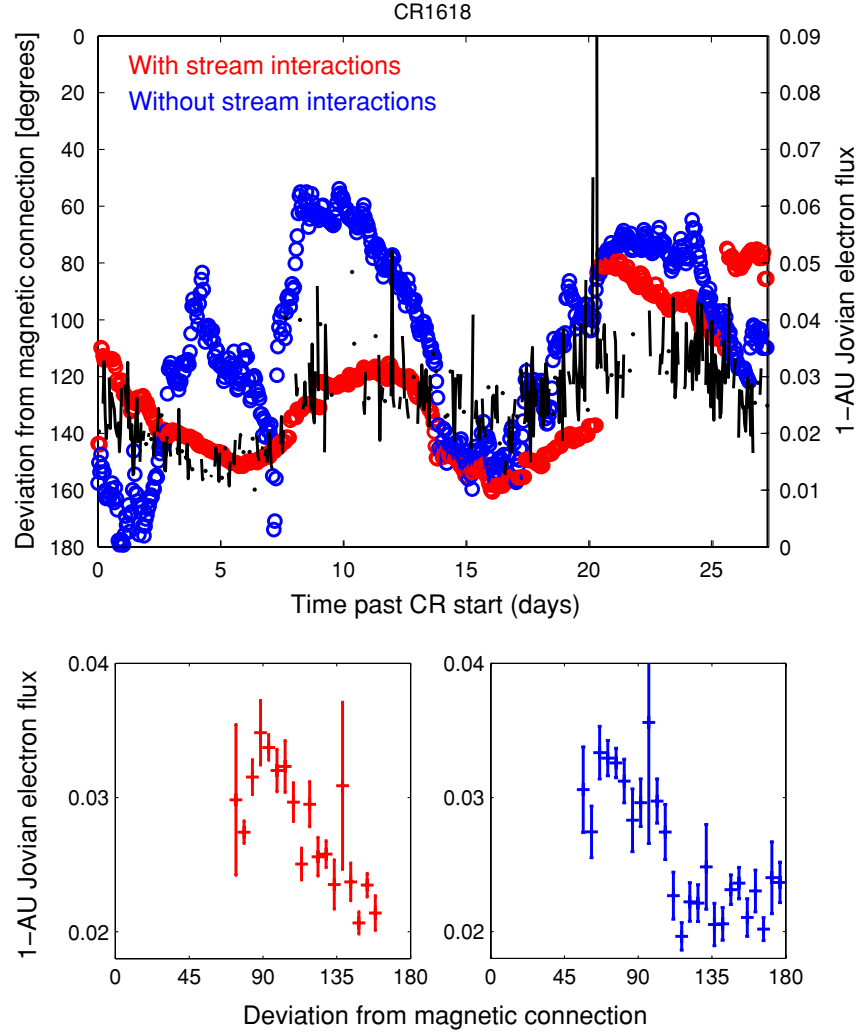

Figure 9. Top panel: the variation of Jovian electron counts near Earth during CR1618, shown as black crosses and the right-hand axis, with estimates of magnetic connectivity to Jupiter. The blue points and the left-hand axis show $\Delta \phi_{\mathrm{PS}}$, the angular separation from Jovian magnetic connection estimated when solar wind stream interactions are not taken into account, whereas the red points show $\Delta \phi_{\mathrm{SI}}$, where stream interactions are included. The bottom panels show the corresponding scatter plots. The correlation between magnetic connectivity and Jovian electron counts is stronger when the effect of stream interactions is included.

(A color version of this figure is available in the online journal.)

Jovian electron counts. Note that quantitative agreement is not expected, as the propagation model was only applied to a single Carrington rotation of data close to solar minimum, whereas the Jovian electron counts are from the analysis of three whole solar cycles of data. Furthermore, the propagation model is known to be overly simplistic in terms of stream interactions and is used here only for demonstration purposes. In particular, the model's assumption of equal momentum exchange between fast and slow streams is apparent in fast wind being under-deflected compared to the Jovian electron peak, which is expected as slow solar wind streams typically exhibit a higher density than fast wind streams. This analysis demonstrates, however, that Jovian electrons in near-Earth space are likely ordered primarily by magnetic connectivity to Jupiter's magnetosphere. The implications of these results are discussed in Section 5.

\section{DISCUSSION}

We have performed a comprehensive survey of Jovian nearrelativistic electrons in near-Earth space over the last three solar cycles. As has been previously established (Chenette 1980; Moses 1987), Jovian electron flux at Earth is ordered by the Jovian synodic period, with the peak "season" occurring during the latter half of the orbit. This has been attributed to the Parker spiral structure of the interplanetary magnetic field leading to an optimum magnetic connection at this time. We 
computed the separation of Earth from an ideal Parker spiral connection to Jupiter using the observed near-Earth solar wind speed and found that while Jovian electron counts are peaked at the expected Parker spiral magnetic connection, there remains significant spread about this point. This is unlikely to be solely the result of strong cross-field diffusion, as slow solar wind streams in near-Earth space show a peak in Jovian electron counts consistent with a magnetic connection strongly underwound from an ideal Parker spiral, whereas fast streams are consistent with an over-wound field.

We then examined a single Carrington rotation in late 1974 during which the solar wind was particularly recurrent in nature, and when Pioneer 11 was upstream of Jupiter. Using a simple ballistic propagation of the near-Earth solar wind parameters, but accounting for stream interactions, the stream and magnetic sector structure at Pioneer 11 can be reasonably well reproduced, indicating that the magnetic field configuration between 1 and $5 \mathrm{AU}$ is also well reconstructed. The under- (over-)winding of the magnetic field suggested by Jovian electron observations in fast (slow) solar wind intervals is found to be consistent with deflections by stream interactions. Thus, while Jovian electrons must undergo significant cross-field diffusion, as they are observed in near-Earth space throughout the Jovian synodic period, we conclude that magnetic connectivity to Jupiter's magnetosphere is likely the primary factor in ordering nearEarth Jovian electron flux (see also Moses 1987). Furthermore, the apparent modulation of Jovian electrons by CIR-associated density enhancements acting as barriers to cross-field diffusion is probably the result of stream interactions altering magnetic connectivity between 1 and 5 AU. Quantification of crossfield diffusion parameters for Jovian electrons in the inner heliosphere (Heber et al. 2007) thus requires accurate models of the dynamic heliospheric magnetic field, as the effect of changing magnetic connectivity on the observed flux must first be removed.

On the basis of the results presented in this study, we suggest that Jovian electrons may provide a means of remote sensing of the heliospheric magnetic field structure between 1 and 5 AU. Consequently, they provide a unique way of validating models of the solar wind and heliospheric magnetic field (e.g., Gosling et al. 1976; Dryer \& Smith 1989; Wang \& Sheeley 2003; Zieger \& Hansen 2008) when in situ observations at suitable heliospheric locations are not available. It remains to be seen whether the short timescale variability in Jovian magnetic connectivity can be probed with Jovian electrons. If Jovian electrons remain associated with flux tubes at these scales, it may be possible to quantify the filamentary flux-tube nature of the solar wind (e.g., Mazur et al. 2000; Borovsky 2008), as well as the increase in flux-tube braiding between 1 and $5 \mathrm{AU}$ due to the on-going effect of turbulence (e.g., Crooker et al. 1999, and references therein). Finally, we note that Jovian electron observations could help resolve the continued debate (e.g., Roberts et al. 2007; Burger et al. 2008) about the systematic under-winding of the heliospheric magnetic field relative to a Parker spiral configuration, resulting from footpoint motion of the magnetic field lines (Fisk \& Schwadron 2001; Murphy et al. 2002; Giacalone \& Jokipii 2004; Owens et al. 2007; Schwadron et al. 2008).

This research at Imperial College London was funded by STFC (UK). We are grateful to the Space Physics Data Facility
(SPDF) and National Space Science Data Center (NSSDC) for OMNI data, and the University of New Hampshire for CRNC data (PI: Dr. C. Lopate). M.O. thanks C. Jackman of Imperial College London for useful discussions.

\section{REFERENCES}

Arge, C. N., \& Pizzo, V. J. 2000, J. Geophys. Res., 105, 10465

Baker, D. N., Higbie, P. R., Belian, R. D., \& Hones, E. W. 1979, Geophys. Res. Lett., 6, 531

Borovsky, J. E. 2008, J. Geophys. Res. (Space Phys.), 113, A08110

Burger, R. A., Krüger, T. P. J., Hitge, M., \& Engelbrecht, N. E. 2008, ApJ, 674, 511

Cargill, P. J. 2004, Sol. Phys., 221, 135

Chenette, D. L. 1980, J. Geophys. Res., 85, 2243

Chenette, D. L., Conlon, T. F., \& Simpson, J. A. 1974, J. Geophys. Res., 79, 3551

Christon, S. P., Chenette, D. L., Baker, D. N., \& Moses, D. 1989, Geophys. Res. Lett., 16, 1129

Conlon, T. F. 1978, J. Geophys. Res., 83, 541

Conlon, T. F., \& Simpson, J. A. 1977, ApJ, 211, L45

Crooker, N. U., et al. 1999, Space Sci. Rev., 89, 179

Dryer, M., \& Smith, Z. K. 1989, Earth Moon Planets, 45, 161

Eraker, J. H., \& Simpson, J. A. 1979, ApJ, 232, L131

Ferreira, S. E. S., Potgieter, M. S., Burger, R. A., Heber, B., \& Fichtner, H. 2001a, J. Geophys. Res., 106, 24979

Ferreira, S. E. S., Potgieter, M. S., Burger, R. A., Heber, B., Fichtner, H., \& Lopate, C. 2001b, J. Geophys. Res., 106, 29313

Fisk, L. A., \& Schwadron, N. A. 2001, ApJ, 560, 425

Giacalone, J., \& Jokipii, J. R. 2004, ApJ, 616, 573

Gosling, J. T., Hundhausen, A. J., \& Bame, S. J. 1976, J. Geophys. Res., 81, 2111

Gosling, J. T., \& Pizzo, V. J. 1999, Space Sci. Rev., 89, 21

Hamilton, D. C., \& Simpson, J. A. 1979, ApJ, 228, L123

Heber, B., et al. 2007, Planet. Space Sci., 55, 1

Henize, V. K., Ferreira, S. E. S., \& Potgieter, M. S. 2003, in Proc. of the 28th Intl. Cosmic Ray Conf., ed. T. Kajita et al. (Tokyo: Universal Academy Press), 3819

Kanekal, S. G., Baker, D. N., Blake, J. B., Looper, M. D., Mewaldt, R. A., \& Lopate, C. A. 2003, Geophys. Res. Lett., 30, 1795

Lheureux, J., \& Meyer, P. 1976, ApJ, 209, 955

Mazur, J. E., Mason, G. M., Dwyer, J. R., Giacalone, J., Jokipii, J. R., \& Stone, E. C. 2000, ApJ, 532, L79

McComas, D. J., Elliott, H. A., Schwadron, N. A., Gosling, J. T., Skoug, R. M., \& Goldstein, B. E. 2003, Geophys. Res. Lett., 30, 1517

Mewaldt, R. A., Stone, E. C., \& Vogt, R. E. 1976, J. Geophys. Res., 81, 2397

Morioka, A., \& Tsuchiya, F. 1996, Geophys. Res. Lett., 23, 2963

Moses, D. 1987, ApJ, 313, 471

Murphy, N., Smith, E. J., \& Schwadron, N. A. 2002, Geophys. Res. Lett., 29, 23

Owens, M. J., Arge, C. N., Spence, H. E., \& Pembroke, A. 2005, J. Geophys. Res., 110, A12105

Owens, M. J., Schwadron, N. A., Crooker, N. U., Hughes, W. J., \& Spence, H. E. 2007, Geophys. Res. Lett., 34, L06104

Owens, M. J., et al. 2008, Space Weather, 6, S08001

Pizzo, V. J. 1991, J. Geophys. Res., 96, 5405

Press, W., Flannery, B., Teukolsky, S., \& Vetterling, W. 1989, Numerical Recipes (Cambridge: Cambridge Univ. Press), 488

Pyle, K. R., \& Simpson, J. A. 1977, ApJ, 215, L89

Roberts, D. A., Giacalone, J., Jokipii, J. R., Goldstein, M. L., \& Zepp, T. D. 2007, J. Geophys. Res., 112, 8103

Schardt, A. W., McDonald, F. B., \& Trainor, J. H. 1983, J. Geophys. Res., 88, 1989

Schwadron, N. A., Owens, M., \& Crooker, N. U. 2008, Astrophys. Space Sci. Trans., 4, 19

Simpson, J. A., Smith, D. A., \& Zhang, M. 1993, J. Geophys. Res., 98, 21129

Teegarden, B. J., McDonald, F. B., Trainor, J. H., Webber, W. R., \& Roelof, E. C. 1974, J. Geophys. Res., 79, 3615

Tsuchiya, F., Morioka, A., \& Misawa, H. 1999, Earth Planets Space, 51, 987

Wang, Y.-M., \& Sheeley, N. R., Jr. 2003, ApJ, 599, 1404

Zieger, B., \& Hansen, K. C. 2008, J. Geophys. Res., 113, A08107 